\title{
LEAVES OF FOLIATIONS WITH A TRANSVERSE GEOMETRIC STRUCTURE OF FINITE TYPE
}

\author{
ROBERT A. WOLAK
}

\begin{abstract}
In this short note we find some conditions which ensure that a $G$ foliation of finite type with all leaves compact is a Riemannian foliation or equivalently the space of leaves of such a foliation is a Satake manifold. A particular attention is paid to transversely affine foliations. We present several conditions such ensure completeness of these foliations.
\end{abstract}

In this short note we shall study leaves of foliations with some transverse geometric structure. In the first section we deal with $G$-foliations of finite type with compact leaves. The main point is to determine whether the space of leaves of such a foliation is a Satake manifold. In the second section we turn our attention to transversely affine foliations.

The question is of importance as, if the space of leaves is a Satake manifold, the foliation itself is Riemannian. It is not a trivial problem. Even for transversely affine foliations with all leaves compact we do not know whether such a foliation is Riemannian. Of course, some additional conditions like completeness or distality are sufficient, but we have been unable to show that a transversely affine foliation with all leaves compact is complete.

The author would like to thank very much indeed Marcel Nicolau for proposing the subject of this note and for many discussions which helped understand bet ter the geometry of foliations in general and the foliations with compact leaves in particular. This note was partially written while the author was a guest of the Centre de Recerca Matematica. He is also very grateful to Professors Manuel Castellet and Joan Girbau for the invitation and the creation of most agreable atmosphere for work. Finally, the author would like to express his deep gratitude to Professor Gilbert Hector whose remarks and advice helped ameliorate a great deal the first version of the paper.

1. Let $\mathcal{F}$ be a $G$-foliation. The bundle $L(M ; \mathcal{F})$ of transverse linear frames admits a foliated reduction $B(M, G ; \mathcal{F})$ to the group $G$. The foliation $\mathcal{F}$ is called of finite type $k$ if the lie group $G$ is of type $k$. This means that the foliation $\mathcal{F}_{k}$ on the total space of the $(k-1)-$ th prolongation $B^{k-1}(M, G ; \mathcal{F})$ of the foliated $G$-structure $B(M, G ; \mathcal{F})$ is a T.P. foliation, cf. [16] and [20]. The leaves of $\mathcal{F}_{k}$ are converings of leaves of $\mathcal{F}$. Therefore the following is true. 
Lemma 1. Leaves of the foliation $\mathcal{F}_{k}$ are holonomy coverings of leaves of $\mathcal{F}$. Thus a leaf of $\mathcal{F}_{k}$ is compact iff the corresponding leaf of $\mathcal{F}$ is compact and has finite holonomy group.

Proof: Let $U=\left\{U_{i}, f_{i}, g_{i j}\right\}$ be a cocycle defining the foliation $\mathcal{F}$ with the following properties:

(i) the open covering $\left\{U_{i}\right\}$ is locally finite,

(ii) the open sets $U_{i}$ are relatively compact,

(iii) the submersions $f_{i}$ take values in a q-manifold $N$ admitting a $G$-structure $B(N, G)$ and have connected fibres,

(iv) the local diffeomorphisms $g_{i j}$ of $N$ are local automorphisms of the $G$ structure $B(N, G)$.

Then the foliation $\mathcal{F}_{k}$ of the total space $B^{k-1}$ of $B^{k-1}(M, G, \mathcal{F})$ is given by a cocycle $\mathcal{U}^{k}=\left\{V_{i}, B^{k-1}\left(f_{i}\right), B^{k-1}\left(g_{i j}\right)\right\}$ where $V_{i}$ is the total space of $B^{k-1}(M, G ; \mathcal{F}) \mid U_{i}, B^{k-1}\left(f_{i}\right)$ is the fibre mapping corresponding to $f_{i}$ of $B^{k-1}(M, G ; \mathcal{F})$ into $B^{k-1}(N, G)$ and $B^{k-1}\left(g_{i j}\right)$ the corresponding local diffeomorphism of the total space $B_{N}^{k-1}$ of $B^{k-1}(N, G)$. The holonomy pseudogroup $\mathcal{H}^{k}$ of the foliation $\mathcal{F}_{k}$ given by the cocycle $\mathcal{U}^{k}$ consists of local diffeomorphisms of $B_{N}^{k-1}$ preserving a parallelism of this manifold, cf. [11], [17], [19]. Therefore, they are determined by their values at any point, and the elements of the holonomy pseudogroup $\mathcal{H}$ generated by the cocycle $\mathcal{U}$ are determined by their $k$-jets. Hence the leaves of $\mathcal{F}_{k}$ are the holonomy coverings of leaves of $\mathcal{F}$. The holonomy group of a leaf $L$ of the foliation $\mathcal{F}$ at a point $x$ of $U_{i}$, up to conjugation, is the group $\boldsymbol{H}_{\underline{x}}=\left\{(h)_{\underline{x}}: h(\underline{x})=\underline{x}, h \in \mathcal{H}\right\}$ where $\underline{x}=f_{i}(x)$ and $(h)_{\underline{x}}$ denotes the germ of the local diffeomorphism $h$ at the point $\underline{x}$. The leaf $L$ is compact iff the $\mathcal{H}$-orbit of $\underline{x}$ is finite. Therefore, the lemma is equivalent to the following one:

an $\mathcal{H}$-orbit of $\underline{x}$ is finite and the group $\mathrm{H}_{\underline{x}}$ is finite iff for any point $\tilde{x}$ over $\underline{x}$ of $B_{N}^{k-1}$ the $\mathcal{H}^{k}$-orbit of $\tilde{x}$ is finite.

The statement that the $\mathcal{H}^{k}$-orbit of $\tilde{x}$ is finite means precisely that the set $\mathrm{H}_{\underline{x}}^{k}=\left\{j_{\underline{x}}^{k} h: h \in \mathcal{H}\right.$ and $\left.\underline{x} \in \operatorname{dom} h\right\}$ is finite. The fact that local automorphisms of the $G$-structure $B(N, G)$ are determined by their $k$-jets ensures that the set $\boldsymbol{H}_{\underline{x}}^{k}$ is finite iff the group $\boldsymbol{H}_{\underline{x}}$ is finite and the $\mathcal{H}$-orbit of the point $\underline{x}$ is finite.

Let us assume that all the leaves of $\mathcal{F}_{k}$ are compact. According to Lemma 1 it is so if the leaves of $\mathcal{F}$ are compact and have finite holonomy. Then the Reeb Stability Theorem (cf. [9]) ensures that the space of leaves $M / \mathcal{F}$ of the foliation $\mathcal{F}$ is a Satake manifold.

The converse is also true. Let $\mathcal{F}$ be a foliation on a compact manifold. If the space of leaves is a Satake manifold the foliation cannot have a non-compact leaf. Otherwise this leaf could not be separated from any other leaf contained in its closure. Moreover, the structure of a Satake manifold ensures that these compact leaves cannot have infinite holonomy. Therefore we have proved the 
following:

Corollary. All the leaves of the foliation $\mathcal{F}_{k}$ are compact iff the space of leaves of the foliation $\mathcal{F}$ is a Satake manifold.

This corollary leads us to the following proposition. We recall that a $G-$ foliation $\mathcal{F}$ of finite type $k$ is transversely complete if the transverse parallelism of the foliation $\mathcal{F}_{k}$ is complete (cf. [16]).

Theorem 1. Let $\mathcal{F}$ be a transversely complete $G$-foliation of finite type $k$ on a compact manifold $M$. All the leaves of $\mathcal{F}$ are compact iff the space of leaves of $\mathcal{F}$ is a Satake manifold.

Proof: The set of points of generic leaves (i.e. without holonomy) is open and dense in $M$ (cf. [8][3]). Thus, according to Lemma 1 the folliation $\mathcal{F}_{k}$ has compact leaves. Moreover, as it is a complete T.P. foliation, all its leaves are compact and the theorem restults from Corollary to Lemma I.

As a corollary we obtain the following fact due to P. Molino (cf. [12], [13]).

Corollary 1. Let $\mathcal{F}$ be a Riemannian foliation on a compact manifold with all leaves compact. Then the space of leaves of $\mathcal{F}$ is a Satake manifold.

Proof: A Riemannian foliation on a compact manifold is a transversely complete foliation of type 1 .

As a corollary of the proof of Theorem 1 we get:

Corollary 2. Let $\mathcal{F}$ be a transversely complete $G$-foliation of finite type. If the foliation $\mathcal{F}$ has a compact leaf with finite holonomy then all the leaves of the foliation $\mathcal{F}$ are compact with finite holonomy and the space of leaves of $\mathcal{F}$ is a Satake manifold.

The assumption of transverse completeness which ensures that all the leaves of $\mathcal{F}_{k}$ are compact can be weakened in many ways. In fact, for us, it is enough to show that the union of the compact leaves of $\mathcal{F}_{k}$ is open and closed, or just closed as it is always open. For example, we have the following.

Proposition 1. Let $\mathcal{F}$ be a $G$-foliation of finite type $k$ with all leaves com pact on a compact manifold $M$. If one of the following conditions is satisfied:

(a) the foliation $\mathcal{F}_{k}$ is a complete Riemannian foliation,

(b) the foliation $\mathcal{F}$ is given by an action of a Lie group $K$ such that:

(i) there exists a supplementary subbundle $Q$ to $T \mathcal{F}$ invariant by $K$,

(ii) the group $K$ acts by automorphisms of the $G$-structure $B(M, G ; \mathcal{F})$, 
then the space of leaves of $\mathcal{F}$ is a Satake manifold.

The proof of the part (a) of the proposition is based on the following lemma for T.P. foliations.

Lemma 2. If a transversely parallelisable foliation $\mathcal{F}$ has a compact leaf and admits a complete bundle-like metric for the foliation $\mathcal{F}$ then the leaves of $\mathcal{F}$ are compact.

Proof: At the very beginning let us stress that there is no connection between the T.P. and the bundle-like metric. Let us pass to the bundle of transverse orthonormal frames. The lifted foliation is a complete T.P. one as the bundlelike metric is complete. It has also a compact leaf. Thus all leaves of this foliation are compact which implies that all leaves of $\mathcal{F}$ are compact.

Part (a) of the proposition is a consequence of Lemmas 1 and 2 and the fact that the foliation has a compact leaf without holonomy.

The proof of the part (b) depends on the fact that under these assumptions the lifted foliation $\mathcal{F}_{k}$ is given by an action of the group $K$ for which there exists a $K$-invariant supplementary subbundle $Q_{k}$ to $\mathcal{F}_{k}$ such that the transverse paralellism consisting of vector fields tangent to $Q_{k}$ is also $K$-invariant. Then integral curves of vector fields of this transverse parallellism are "uniformly" defined along the leaves. This property ensures that if one leaf of $\mathcal{F}_{k}$ is compact so are all the leaves.

Remarks 1 . The assumption that the manifold $M$ is compact and that all the leaves of the foliation $\mathcal{F}$ are compact can be replaced by "there exists a compact leaf with finite holonomy".

2. If the group $K$ of (b) is compact a $K$-invariant subbundle $Q$ supplementary to $T \mathcal{F}$ always exists.

3. The above considerations are also valid for $\nabla-G$-foliations as the foliation $\mathcal{F}_{1}$ of the total space of $B(M, G ; \mathcal{F})$ is T.P. More generally, it is also true for foliations admitting foliated Cartan connections (cf. [1]).

4. Lemma 2 is the weakest possible as indicates the following example:

Example. Let $T^{2}=S^{1} \times S^{1}$ be the 2-torus foliated by circles $\{x\} \times S^{1}$. When we puncture it, we obtain a T.P. foliation of the punctured torus whose all but one leaves are compact. None of the bundie-like metrics for this foliated manifold can be complete.

Now, let us restrict our attention to $\nabla-G$-foliations. Using the exponential mapping defined by transverse geodesics of the transversely projectable connection we can try to linearize the holonomy of any leaf $L$ of the foliation, i.e. we would like to know whether there exists an open neighbourhood $D$ of $L$ in the normal bundle $N(L)$ of the leaf $L$ and an open saturated neighbourhood $U$ of $L$ in $M$ such that exp $D: D \rightarrow M$ is a diffeomorphism of $D$ onto $U$. If it is true, then for any point $x \in L$ and any element $h$ of the holonomy group of $L$ 
at $x$ we have: $\exp \cdot h \cdot(\exp \mid D)^{-1}\left|D_{x}=d_{x} h\right| D_{x}$. Let us assume that we can linearize holonomy. If the leaves are compact, then any orbit of the linear holonomy group is finite. In this case it is not difficult to see that any element of the linear holonomy group is of finite order and the Schur Theorem (cr. [2]) ensures that this group is finite and that the space of leaves of $\mathcal{F}$ is a Satake manifold.

The converse is, in fact, also true. The results of D.B.A. Epstein (cf. [4])ensure that any leaf has a basis of open saturated neighbourhoods. As the leaves are compact, for any leaf $L$ there exists an $\varepsilon>0$ such that $\exp \mid D_{\varepsilon}(L)$ : $D_{\varepsilon}(L) \rightarrow M$ is a diffeomorphism onto the image, where $D_{\epsilon}(L)=\{v \in N(L)$ : $\|v\|<\varepsilon\}$ for some Riemannian metric on $N(\mathcal{F})$. Then we can find an open saturated neighbourhood $U$ of $L$ contained in $\exp \left(D_{\varepsilon}(L)\right)$. Thus exp | $\exp ^{-1}(U)$ defines the required linealization of the holonomy group of $L$. We have proved the following theorem.

Theorem 2. Let $\mathcal{F}$ be a $\nabla-G$-foliation with all leaves compact. Then the holonomy of any leaf of $\mathcal{F}$ can be linearized iff the space of leaves of $\mathcal{F}$ is a Satake manifold.

As a corollary we get the fact that the space of leaves of a Riemannian foliation with all leaves compact on a non-compact manifold is a Satake manifold. It is so since the holonomy of any compact leaf of a Riemannian foliation is linearisable.

These considerations can be summarized as follows:

Corollary 1. Let $\mathcal{F}$ be a $\nabla-G$-foliation with all leaves compact on a compact manifold $M$. Then the following conditions are equivalent:

(1) the holonomy of each leaf is linearisable,

(2) $\mathcal{F}$ is Riemannian,

(3) the holonomy of each leaf if fnite,

(4) the space of leaves is a Satake manifold,

(5) the volume function is locally bounded,

(6) the foliation $\mathcal{F}$ is minimal.

Corollary 1 is a consequence of our considerations and results of $[3],[4]$ and [14], see also Appendix B by V. Sergiescu in [13]. Conditions (2), (3), (4), (5) and (6) are equivalent for any foliation with all leaves compact on a compact manifold.

Corollary 2. If the foliation $\mathcal{F}$ is of codimension 2, then the holonomy of any leaf is linearisable.

Proof: It is a consequence of Theorem 2 of [3]. 
2. In this section we shall study in detail transversely affine foliations. A transversely affine foliation $\mathcal{F}$ is given by a cocycle $\mathcal{U}=\left\{U_{i}, f_{i}, g_{i j}\right\}$ with values in an affine space $E$ for which the transformations $g_{i j}$ are the restrictions of elements of the affine group. Therefore such a foliation is a $\nabla-G$-foliation. A transversely affine foliation is developable (cf. [7]). Let $\hat{M}$ be the holonomy covering of $M$ and $D: \hat{M} \rightarrow E$ the developing mapping. Then each leaf of the lifted foliation $\hat{\mathcal{F}}$ of $\hat{M}$ is the holonomy covering of the corresponding leaf of $\mathcal{F}$. We call the corresponding homomorphism $\alpha: \pi_{1}(M) \rightarrow$ Aff $(E)$ the affine holonomy and its image the affine holonomy group. The homomorphism $\lambda: \pi_{1}(M) \rightarrow G L(E)$ assigning to each class $[\sigma]$ the linear part of $\alpha([\sigma])$ is called the linear holonomy and its image the linear holonomy group. It is not difficult to prove (cf. [18]) that if the foliation $\mathcal{F}$ is transversely complete (some would say geodesically complete) then the developing mapping is a trivial bundle with the fibre being the holonony covering of leaves of $\mathcal{F}$. For many problems this property is sufficient. Therefore we say that a transversely affine foliation $\mathcal{F}$ is complete if the developing mapping $D: \hat{M} \rightarrow E$ is a trivial bundle over $E$.

The results of [6] proved for affine manifolds can be generalized without many difficulties to the case of transversely affine foliations. In fact, the following theorem holds (we assume the notation and definitions of [6]).

Theorem 3. Let $M$ be a compact manifold and $\mathcal{F}$ a transyersely affine foliation on $M$ whose affine holonomy group is nilpotent. Then the following conditions are equivalent:

(a) $\mathcal{F}$ is complete,

(b) the developing mapping is surjective,

(c) the linear holonomy is unipotent,

(d) the affine holonomy is irreducible,

(e) the affine holonomy is indecomposable.

It is not difficult to see that most theorems have their corresponding versions for transversely affine foliations. In general, the terms "vector field" and "form" on the manifold $M$ are replaced by "foliated vector field" and "base-like form" on the foliated manifold $(M, \mathcal{F})$. We leave to the reader the statements and proofs of the theorems for transversely affine foliations corresponding to the following ones of $[6]$ : $3.2-3,4.1,4.3-4,6.1-4,6.6,6.8-9$. We shall only prove the following (ck. Theorem 2.2, [6]).

Theorem 4. Let $\mathcal{F}$ be a complete transversely affine foliation on a compact manifold $M$. Then the affine holonomy representation is irreducible.

Proof: The manifold $M$ is the quotient of $\hat{M}$ by the group $\pi$ of the deck transformations. Thus $M=\hat{L} \times E \mid \pi$ and the group $\pi$ is isomorphic to the affine holonomy group. Let us assume that $F$ is an affine subspace of $E$ invariant under $\pi$. Then the inclusion of $\hat{L} \times F \mid \pi$ into $\hat{L} \times E\} \pi$ induces isomorphisms 
of the homotopy groups and therefore it is a homotopy equivalence of compact manifolds $\hat{L} \times F \mid \pi$ and $M$. Thus they must be of the same dimension. Hence $F=E$ and the affine holonomy representation is irreducible.

As a consequence of Theorem 4 we get:

Corollary. Let $\mathcal{F}$ be a transversely affine foliation with nilpotent holonomy group of a compact manifold $M$ with all leaves compact. Then, if one of the conditions of Theorem $s$ is satisfied, the space of leaves of the foliation $\mathcal{F}$ is a Satake manifold.

Proof: Theorem 3 ensures that the holonomy covering $\hat{M}$ is a product $\hat{L} \times E$. But, since the generic leaf of $\mathcal{F}$ is compact, so is $\hat{L}$. This assures that the foliation is (transversely) geodesically complete. In fact, let us take any subbundie $Q$ supplementary to $T \mathcal{F}$. Then the lifted bundle $\hat{Q}$ on $\hat{M}$ is a horizontal bundle for the developing mapping. As the fibre is compact, we can lift any curve in $E$ to a curve tangent to $\hat{Q}$ on $\hat{M}$. The projections on $M$ of the lifts of straight lines are the geodesics tangent to the bundle $Q$ of the transversely projectable connection. Then an application of Theorem 1 completes the proof.

In the proof of Corollary we have actually demonstrated that a complete transversely affine foliation with all leaves compact is geodesically complete.

Proposition 2. Let $\mathcal{F}$ be a complete transversely affine foliation on a compact manifold with all leaves compact. Then $\mathcal{F}$ is geodesically complete.

Now, we shall turn our attention to a more specific class of transversely affine foliations. The flat connection on $E\left(R^{q}\right)$ defines a transversely projectable connection $\nabla$ in the normal bundle of the foliation. We can consider its linear holonomy: for any point $x$ of $M$ there is a homomorphism $h_{x}: \pi_{1}(M, x) \rightarrow$ $G L(q)$. When $M$ is connected, by changing the point $x$ we get conjugate homomorphisms. For simplicity's sake we identify the normal bundle $N(M, \mathcal{F})$ with a subbundle $Q$ of $T M$ supplementary to $T \mathcal{F}$.

We say that the foliation $\mathcal{F}$ is distal if the action of the linear holonomy group $H_{x}=i m h_{x}$ on $R^{q} \cong Q_{x}$ is distal (cf. [2], [5]). This condition does not depend on the choice of a point $x$. The condition equivalent to the distality ensures that the group $H_{x}$ preserves a flag of subspaces $V_{0}=\{0\} \subset V_{1} \cdots \subset V_{k+1}=$ $Q_{x}$. Since the connection $\nabla$ is without torsion the corresponding subbundles $T \mathcal{F} \subset Q_{1} \subset \ldots Q_{k} \subset T M$ of the tangent bundle are involutive, thus they define a flag of foliations $\mathcal{F}_{0}=\mathcal{F} \subset \mathcal{F}_{1} \subset \ldots \mathcal{F}_{k}$. The foliations $\mathcal{F}_{i}, i=1, \ldots, k$ are totally geodesic with respect to the connection $\nabla$. Moreover, we can find a Riemannian metric on $M$ adapted to the flag $\left(\mathcal{F}_{0}, \mathcal{F}_{1}, \ldots \mathcal{F}_{k}\right)$ such that the foliations of leaves of $\mathcal{F}_{i+1}$ by leaves of $\mathcal{F}_{i}$ are Riemannian (cf. [5]). The foliations $\mathcal{F}_{i}$ correspond via the developing mapping to the foliations $\mathcal{F}_{i}^{0}$ of $R^{q}$ which are totally geodesic with respect to the flat connection of $R^{q}$. Thus 
these foliations are by parallel affine subspaces of $R^{q}$. From this fact it results that the foliations of leaves of $\mathcal{F}_{i+1}$ by leaves of $\mathcal{F}_{i}$ are complete Riemannian foliations modelled on $R^{g_{i}}$ where $q_{i}$ is the codimension of $\mathcal{F}_{i}$ in $\mathcal{F}_{i+1}$. Let $D: \hat{M} \rightarrow R^{q}$ be the developing map and $\hat{\mathcal{F}}_{i}$ be the lift of $\mathcal{F}_{i}$ to $\hat{M}$. Let $L_{i+1}$ be a leaf of $\mathcal{F}_{i+1}^{\hat{n}}$ and $L_{i}$ a leaf of $\hat{\mathcal{F}}_{i}$ contained in $L_{i+1}$. The foliation of $L_{i+1}$ by leaves of $\hat{\mathcal{F}}_{i}$ is a complete Riemannian foliation modelled on $R^{q_{i}}$ given by a global submersion $D_{i}: L_{i+1} \rightarrow R^{q_{i}}$ defined as follows

$$
L_{i+1} \rightarrow R^{\tilde{q_{i}}} \rightarrow R^{q_{i}} \cong R^{\tilde{q_{i}}} / R^{\tilde{q_{i-1}}}
$$

where $\tilde{q}_{i}=\Sigma_{j=0}^{i} q_{j}$.

A well-known Herman's theorem (cf. [10]) ensures that $D_{i}$ is a locally trivial fibre bundle, and as $R^{q_{i}}$ is contractible it is a trivial fibre bundle, i. e. $L_{i+1} \cong$ $L_{i} \times R^{q_{i}}$, and therefore $\hat{M} \cong \hat{L} \times R^{q}$ where $\hat{L}$ is a leaf of the lifted foliation $\hat{\mathcal{F}}$. We have just proved the following proposition:

Proposition 3. A distal transversely affine foliation on a compact manifold is complete.

This with Proposition 2 gives the following:

Theorem 5. Let $\mathcal{F}$ be a transversely affine foliation on a compact manifold $M$. If the foliation $\mathcal{F}$ is distal and all the leaves are compact, then the space of leaves of $\mathcal{F}$ is a Satake manifold.

The final remarks. Our considerations lead to pose some more generat questions.

1) When a $G$-foliation is a Riemannian one?

In general, for foliations with all leaves compact the answer is: when the space of leaves is a Satake manifold, cf. Corollary 1 to Theorem 2 . In the case of $G$-foliations with all leaves compact the completeness of this foliation is sufficient, cf. Theorem 1 . Theorem 2 ensures that for $\nabla-G$-foliations it is sufficient to know that the holonomy of leaves is linearisable. Corollary to Theorem 3 and Theorem 5 provide answers for particular classes of transversely affine foliations.

We have looked at this question from a totally different point of view in [21].

2) Does there exist a transversely affine foliation with all leaves compact which is not Riemannian?

We do not know the answer yet, but Theorem 5 indicates that we have to look for non-distal discrete subgroups of Aff $(E)$. 


\section{References}

1. R.A. Blumenthal, Cartan connections in foliated bundles, Mich. Math. J. 31 (1984), 55-63.

2. J.P. CONZE, Y. GUIVARC'H, Remarques sur la distalité dans les espaces vectoriels, C.R. Acad. Sci. Paris 278 (1974), 1083-1086.

3. R. EDWARD, K. MILleET, D. Sullivan, Foliations with all leaves compact, Topology 16 (1977), 13-32.

4. D.B.A. EPSTEIN, Foliations with all leaves compact, Ann. Inst. Fourier 26, 1 (1976), 265-282.

5. D. FRIED, Distality, completeness and affine structures, J. Diff. Geom. 24 (1986), 265-273.

6. D. FRIED, W.M. GOLdMAN, M.W. HIRSCH, Affine manifolds with nilpotent holonomy, Comm. Math. Helv. 56 (1981), 487-523.

7. W.M. GOLDMAN, M.W. HIRSCH, G. LEVITT, Invariant measures for affine foliations, Proc. AMS 86, 3 (1982), 511-518.

8. G. HECTOR, "Feuilletages en cylindres," in "Geometry and Topology", Rio de Janeiro 1976, Springer LN, 1977, pp. 252-270, 597.

9. G. HECTOR, U. HIRSCH, Introduction to the Geometry of Foliations, $A-B$ Vieweg (1983).

10. R. HERMAN, A sufficient condition that a mapping of Riemannian manifolds be a fibre bundle, Proc. AMS 11 (1960), 232-242.

11. S. KoBAYASHI, "Transformation Groups in Geometry," Springer, Ergebnisse de Mathematik, 1972.

12. P. Molino, Géométrie global des feuilletages riemanniens, Proc. Kon. Neder. Akad. 85 (1982), 45-76.

13. P. Molino, Riemannian Foliations, Progress in Math. 73 (1988), Birkhäuser.

14. H. RUMMLER, Quelques notions simples en géométrie riemannienne et leurs applications aux fetilletages compacts, Comm. Math. Helv. 54 (1979), 224-239.

15. R. Wolak, On $\nabla-G$-foliations, Suppl. Rend. Cir. Mat. Palermo 6 (1984), 329-341.

16. R. Wolak, On G-foliations, Ann. Pol. Math. 46 (1985), 371-377.

17. R. WOLAK, On transverse structures of foliations, Suppl. Rend. Cir. Mat. Palermo 9 (1985), 227-243.

18. R. WOLAK, Foliations admitting transverse systems of differential equations, Comp. Math. 67 (1988), 89-101.

19. R. WoLAK, Foliated and associated geometric structures on follated manifolds, preprint $C R M$, Barcelona no. 60 . 
20. R. WOLAK, The structure tensor of a transverse $G$-structure on a foliated manifold, preprint 1988.

21. R. WOLAK, $G$-foliations with the central sheaf of compact type, preprint $C R M$, Barcelona no. 58.

\author{
Robert A. Wolak: Instytut Matematyki \\ Uniwersytet Jagiellonski \\ Wl. Reymonta 4 \\ 30-059 Krakow \\ POLAND \\ Current address: Universidade de Santiago \\ Departamento de Xeometria e Topoloxia \\ Facultade de Matematicas \\ 15705 Santiago de Compostela \\ SPAIN
}

Rebut el 6 d'octubre de 1988 